\title{
Riesgo cardiovascular relacionado con el consumo de alcohol
}

\author{
Cardiovascular risk associated with alcohol consumption \\ Risco cardiovascular associado com o consumo de álcool
}

\author{
Dr. Jaime E. Cedeño-Zambrano, Dra. Paula A. Vásquez-Jaramillo, Dra. Vanessa E. Roca-Lino \\ jecz23@hotmail.com, paulavj89@hotmail.com, dravanessarocal@gmail.com \\ Universidad Laica "Eloy Alfaro” de Manabí, Manta, Ecuador
}

Recibido: 1 de junio de 2016

Aceptado: 12 de septiembre de 2016

\section{Resumen}

El consumo de alcohol tiene un comportamiento ambivalente sobre el sistema cardiovascular con efectos tanto beneficiosos como nocivos. En esta revisión se indago sobre los efectos del consumo de alcohol tiene el sistema cardiovascular, su patogenia y las pautas de conducta a adoptar respecto a la situación de riesgo cardiovascular. Podemos dar por concluido que el consumo excesivo de alcohol, puede ocasionar miocardiopatía alcohólica (el corazón se dilata y deja de funcionar adecuadamente). También puede generar infartos al corazón. Su consumo regular eleva los niveles de sodio y calcio en la célula y puede producir hipertensión arterial. Cuando se reduce el consumo en personas con hipertensión se logra disminuir la enfermedad. La ingesta aguda de alcohol, puede producir arritmias graves en personas sanas.

Palabras clave: alcohol, corazón, sistema cardiovascular, factores de riesgo.

\section{Abstract}

The consumption of alcohol has an ambivalent behavior on the cardiovascular system with both beneficial and harmful effects. In this review I investigate on the effects of the consumption of alcohol it has on the cardiovascular system, his pathogeny and the guidelines of conduct to adopt with regard to the situation of cardiovascular riskThe consumption of alcohol has an ambivalent 
behavior on the cardiovascular system with both beneficial and harmful effects. In this review I investigate on the effects of the consumption of alcohol it has on the cardiovascular system, his pathogeny and the guidelines of conduct to adopt with regard to the situation of cardiovascular risk. We can give concluded that the excessive consumption of alcohol, can cause miocardiopatía alcoholic (the heart expands and allows working adequately). Also it can generate hearts attacks to the heart. His regular consumption raises the levels of sodium and calcium in the cell and can produce arterial hypertension. When the consumption diminishes in persons with hypertension it is achieved to diminish the disease. The sharp ingestion of alcohol, can produce serious arrhythmias in healthy persons.

Key words: alcohol, heart, cardiovascular system, factors of risk.

\section{Resumo}

O consumo de álcool tem um comportamento ambivalente sobre o sistema cardiovascular com ambos os efeitos positivos e negativos. Nesta revisão foram investigados os efeitos do álcool tem o sistema cardiovascular, sua patogênese e os padrões de comportamento a adoptar em relação à situação de risco cardiovascular. Podemos escrever concluiu que o consumo excessivo pode causar cardiomiopatia alcoólica (coração se expande e não funcionar adequadamente). Você também pode gerar ataques cardíacos. O consumo regular aumenta os níveis de sódio e de cálcio na célula e pode provocar tensão arterial elevada. Quando o consumo é reduzido com hipertensão reduzir a doença é conseguido. ingestão alcoólica aguda, pode produzir arritmias graves em pessoas saudáveis.

Palavras chave: álcool, coração, sistema cardiovascular, factores de risco.

\section{Introducción}

En organismos unicelulares, todos los procesos vitales ocurren en una sola célula. Conforme progresó la evolución de los organismos multicelulares, varios grupos celulares se organizaron en tejidos y órganos con funciones particulares. En seres humanos y otros animales vertebrados los grupos celulares especializados incluyen un aparato digestivo para la digestión y absorción de alimentos, un aparato respiratorio para la captación de $\mathrm{O} 2$ y eliminación de $\mathrm{CO} 2$; un aparato urinario para eliminar productos de desecho metabólico, un aparato cardiovascular para la distribución de nutrimentos, $\mathrm{O} 2$, y productos del metabolismo; un aparato reproductor para perpetuar a la especie; un aparato endocrino y el sistema nervioso para coordinar e integrar la función de los otros aparatos y sistemas. (1) 
El sistema circulatorio posee como función, el distribuir los nutrientes, oxígeno a las células y recoger los desechos metabólicos que se han de eliminar después por los riñones, en la orina, y por el aire exhalado en los pulmones, rico en dióxido de carbono (CO2). De toda esta labor se encarga la sangre, que está circulando constantemente. Además, el aparato circulatorio tiene otras destacadas funciones: interviene en las defensas del organismo, regula la temperatura corporal. (2)

La enfermedad cardiovascular es una de las principales causas de muerte a nivel internacional y se desarrolla por un proceso multifactorial. La mayoría de las enfermedades cardiovasculares son secundarias a la enfermedad ateroesclerótica, por un proceso degenerativo de las arterias que es inducido por el estrés oxidativo y un estado crónico de inflamación. Los factores de riesgo de esta enfermedad son el hábito de fumar, diabetes mellitus, hipertensión arterial, las anormalidades en los niveles de colesterol total y sus fragmentos en el suero, el sobre peso/obesidad, historia familiar de enfermedad cardiovascular precoz y la inactividad física, entre otros. (3)

\section{Factores de riesgo cardiovascular}

Las enfermedades cardiovasculares pueden manifestarse de muchas formas: presión arterial alta, enfermedad arterial coronaria, enfermedad valvular, accidente cerebrovascular y arritmias (latidos irregulares). Según la Organización Mundial de la Salud, las enfermedades cardiovasculares causan el fallecimiento de más de 17 millones de personas en el mundo cada año y es responsable de la mitad de las muertes que ocurren en los Estados Unidos. (4)

La enfermedad arteria coronaria, la forma más frecuente de enfermedad cardiovascular, es la principal causa de muerte en los Estados Unidos en la actualidad. El cáncer, que ocupa el segundo lugar, causa un número de fallecimientos que es apenas un poco más de la mitad de las muertes ocasionadas por enfermedades cardiovasculares. (4)

En los Estados Unidos, más de 80 millones de personas sufren alguna forma de enfermedad cardiovascular. Sin embargo, gracias a muchos estudios en los que participaron miles de pacientes, los investigadores han identificado ciertas variables que desempeñan papeles importantes en las

probabilidades de que una persona presente enfermedades cardiovasculares. Estas variables se conocen como factores de riesgo. (4)

Los factores de riesgo se dividen en dos categorías: principales y secundarios. Los factores de riesgo principales son factores cuya intervención en el riesgo de sufrir enfermedades cardiovasculares se ha 
comprobado. Los factores de riesgo secundario pueden elevar el riesgo de sufrir estas enfermedades. (4)

Cuantos más factores de riesgo tenga una persona, mayores serán sus probabilidades de padecer una enfermedad del corazón. Algunos factores de riesgo pueden cambiarse, tratarse o modificarse y otros no. Pero el control del mayor número posible de factores de riesgo, mediante cambios en el estilo de vida y/o medicamentos, puede reducir el riesgo cardiovascular. (4)

Entre los principales factores de riesgo tenemos: presión arterial alta, colesterol elevado, diabetes, obesidad y sobrepeso, tabaquismo, inactividad física, sexo, herencia, estrés, hormonas sexuales, anticonceptivos orales, y el alcohol.

\section{Consumo de alcohol y riesgo cardiovascular}

Las bebidas alcohólicas son bebidas que contienen etanol (alcohol etílico). La dependencia de las bebidas alcohólicas se denomina alcoholismo. El alcohol es una sustancia psicoactiva o droga, cuyo consumo desmedido puede hacer daño al organismo (básicamente el sistema nervioso o el hígado), además es agente causal indirecto de problemas en la familia y en la sociedad. (5)

Atendiendo a la elaboración se pueden distinguir entre bebidas producidas por fermentación alcohólica (vino, cerveza, hidromiel, sake) en las que el contenido en alcohol no supera los 18-20 grados, y las producidas por destilación, generalmente a partir de un producto de fermentación (licores, aguardientes, etc.). (5)

El alcohol se considera una droga porque puede generar al menos uno de estos tres fenómenos: DEPENDENCIA, TOLERANCIA y/o SINDROME DE ABSTINENCIA. (5)

Cabe señalar que el efecto físico del alcohol no está expuesto a ningún proceso de digestión por lo que en su mayoría pasa primero al intestino delgado para después ser absorbido por el torrente sanguíneo. Sólo una pequeña parte llega directamente a la sangre a través de las paredes estomacales. En la sangre el alcohol es metabolizado (descompuesto para ser eliminado o aprovechado por el organismo) mediante el proceso de oxidación. Es decir, se fusiona con el oxígeno y se descompone de modo que sus elementos básicos abandonan el cuerpo de forma de bióxido de carbono y agua. El primer lugar de oxidación es el hígado, el cual descompone aproximadamente el $50 \%$ del alcohol ingerido en una hora. El resto permanece en el torrente sanguíneo hasta ser eliminado lentamente. (5) 
En la literatura revisada enuncia que el alcohol es un tóxico multisistémico con efectos, tanto agudos como crónicos, claramente nocivos sobre muchos órganos y sistemas de nuestro organismo. (6) Contrariamente, el consumo de alcohol es un hábito social y cultural tan ampliamente extendido en nuestra sociedad occidental que podría considerarse como propio de su misma cultura y costumbres. Al considerar los efectos que el consumo de alcohol ejerce sobre la salud, y en concreto sobre el riesgo de padecer enfermedades cardiovasculares, ha existido siempre una clara dualidad. Por una parte, son obvios sus efectos nocivos cuando se consume con desmesura a altas dosis (efecto tóxico). En este sentido ya Hipócrates en el siglo IV,a.C. recomendaba evitar el consumo de alcohol a los pacientes con hidropesía, al observar que mejoraba su cuadro de insuficiencia cardíaca cuando evitaban su consumo. Desde entonces han pasado muchos años hasta que se han podido constatar de forma objetiva las bases fisiopatológicas del daño orgánico producido por el alcohol sobre el sistema cardiovascular. Se reconoce ampliamente el efecto tóxico que ejerce sobre este sistema cuando se consume en altas dosis. Al reflexionar los efectos nocivos sobre el sistema cardiovascular del consumo de alcohol, cabe diferenciar entre efectos agudos y crónicos. Los primeros suceden cuando se consume alcohol en dosis elevadas (superiores a 60 gramos por ingesta). En cambio, los efectos crónicos se producen por un consumo acumulativo, dosis dependiente en el que juegan tanto la dosis diaria consumida como el tiempo total de consumo. (6)

Por otro lado, siempre ha existido una evidencia, cada vez más objetiva, de que el alcohol ejerce algunos efectos beneficiosos sobre el sistema cardiovascular, sobre todo cuando se consume en bajas dosis, lo que se ha denominado Òefecto tónicoÓ del alcohol. Estas evidencias han sido corroboradas recientemente en numerosos trabajos científicos. Así, desde 1970 más de 100 estudios han observado una asociación epidemiológica entre consumo de alcohol en dosis bajas y mejoría de riesgo o mortalidad cardiovascular. (7)

Varios estudios plantean que aunque el consumo excesivo de alcohol es indiscutiblemente dañino, la evidencia epidemiológica ha señalado que el consumo moderado de alcohol está inversamente asociado con los factores de riesgo cardiovasculares y los eventos cardiovasculares. (8) Sin embargo, después de la descripción de la "paradoja francesa" hace más de 20 años, una parte de las investigaciones se enfocaron, en los diferentes efectos del consumo de vinos comparados con otras bebidas alcohólicas. La paradoja francesa informó la disminución de la incidencia de enfermedad cardiovascular y su mortalidad de la población francesa a pesar de su dieta rica en grasas saturadas. Este fenómeno fue atribuido al consumo de vino y no solo al alcohol, desde que las concentraciones 
de HDL-colesterol del plasma (HLDc) eran similares a las informadas en otros países con un mayor predominio ECV. (3) Algunos detractores han planteado que en lugar del vino tinto, esta paradoja pudiera ser explicada por el incremento del tiempo entre el aumento de las concentraciones de colesterol en suero, y el desarrollo de la placa ateromatosa en las arterias coronarias y riesgo de muerte por enfermedad isquémica del corazón, debido a que la población francesa había disminuido de grasa animal desde la década anterior al estudio. Otros autores creyeron que no solo el vino tinto, sino que también el consumo del alcohol serían responsable de la paradoja. $(9,3)$ A pesar de esta controversia, la paradoja francesa activó extensos estudios acerca de los efectos de los componentes del vino tinto (más concretamente los poli fenoles y sobre todo el resveratrol) para explicar esta paradoja se llevó a una distinción de los tipos de bebidas alcohólicas (con más o menos compuestos fenólicos) y se abrió el debate acerca de cuál tipo de bebida alcohólica es más cardio protectora. (3) Ante la disyuntiva sobre la conveniencia de beber o no beber alcohol, por su influencia como factor de riesgo cardiovascular, es importante dilucidar si existe un límite o un umbral de dosis a partir del cual se pudiera disponer de una dosis segura para poder obtener los efectos beneficiosos del alcohol sin llegar a tener riesgo de sus efectos nocivos.

El presente trabajo aborda tanto los efectos nocivos como los beneficiosos que el alcohol ejerce sobre diferentes esferas del sistema cardiovascular con la finalidad de aportar datos objetivos basados en las evidencias científicas disponibles que nos permitan construir una idea global del espectro de afectación que produce el alcohol y el riesgo cardiovascular específico que comporta.

\section{Cuantificación de la ingesta alcohólica}

Uno de los factores que puede diferenciar entre efecto beneficioso o perjudicial del consumo de alcohol es la dosis consumida. Por ello es muy importante valorar correctamente el consumo realizado tanto de forma aguda como de forma crónica y hablar en términos cuantitativos que sean claros y comparables. La manera más objetiva sería la valoración por gramos de alcohol puro, consumido por unidad de tiempo. (6)

Se considera AD: altas dosis de consumo de alcohol (> 60 g/día); BD: bajas dosis de consumo de alcohol (<20 g/día en mujeres y $<40$ g/día en hombres); DTA: dosis total acumulada de consumo de alcohol a lo largo de la vida del paciente; AVC: accidente vascular cerebral. (6)

Los efectos agudos relacionados con las altas dosis (AD) tenemos: disminución de la contractilidad miocárdica, inducción de arritmias y muerte súbita. (6) 
Con respecto a los efectos crónicos podemos relacionar: incremento de mortalidad global (AD), disminución de mortalidad global (BD), incremento de mortalidad cardiovascular (AD), disminución de mortalidad cardiovascular (BD), disminución de eventos coronarios (BD), reducción de episodios de insuficiencia cardíaca (BD), inducción de hipertensión arterial (efecto dosis-dependiente a partir de 20 g al día), mejoría de arteriopatía periférica (BD), incremento de AVC isquémicos (AD), disminución de AVC isquémicos (BD), aumento de AVC hemorrágicos (AD), desarrollo de miocardiopatía alcohólica (efecto acumulativo en relación con DTA), fase sub clínica con disfunción diastólica y sistólica, insuficiencia cardíaca, arritmias, y muerte súbita. (6)

\section{Efectos agudos del alcohol sobre el sistema cardiovascular.}

El consumo agudo de altas dosis induce a nivel sistémico, la tan conocida intoxicación alcohólica aguda, con depresión progresiva del nivel de consciencia, ataxia y disfunción cortical. En algunos casos se puede desarrollar una miopatía aguda con rabdomiólisis tóxica e inducción de insuficiencia renal aguda por mioglobinuria. Pero también puede inducir muchos efectos cardiovasculares. Los principales son el desencadenamiento de crisis hipertensivas, la depresión de contractibilidad miocárdica y la inducción de arritmias, con posibilidad de muerte súbita. (6)

\section{Efectos sobre la contractilidad cardíaca}

Estudios experimentales realizados con fibras miocárdicas aisladas han puesto de manifiesto que el etanol ejerce un efecto inotrópico negativo, que es dependiente de la dosis y rápidamente reversible al eliminarlo. Así, a los 5 minutos de retirar la exposición a etanol se comprueba una total normalización de la contractilidad cardíaca. Sin embargo, los efectos in vivo a animales de experimentación y humanos han sido muy dispares. Ello puede ser debido al efecto cronotropo positivo por la liberación de catecolaminas que el etanol puede conllevar y que enmascararía su efecto inotrópico negativo. Por ello se tuvo que objetivar este efecto en corazones desnervados o mediante un bloqueo del sistema autonómico con propranolol o atropina. En estas últimas condiciones se mantiene el efecto inhibidor de la contractilidad miocárdica del etanol Se trata de un efecto dosis-dependiente y mediado por la disminución de la entrada de calcio citosólico, que afecta secundariamente a la despolarización de las fibras miocárdicas. Se ha demostrado que este efecto tiene poca relevancia en personas abstemias o en alcohólicos con función cardíaca normal, pero en 
los pacientes con miocardiopatía alcohólica o con cardiopatía de diferentes etiologías una ingesta aguda de altas dosis de etanol puede desencadenar una insuficiencia cardíaca. (6)

\section{Alcohol e insuficiencia cardiaca}

Las principales causas de insuficiencia cardiaca son la cardiopatía isquémica (angina de pecho e infarto de miocardio) y una hipertensión mal controlada prolongada en el tiempo. También destacar, aunque no son las causas más importantes en la aparición de insuficiencia cardiaca, el efecto negativo del consumo excesivo de alcohol (que daña las fibras musculares del corazón), un estrechamiento de las válvulas cardiacas, hipotiroidismo e infección del músculo cardiaco. (10)

\section{Riesgo de arritmia}

El etanol puede modificar las propiedades electrofisiológicas del corazón, alterando el tiempo de conducción y los períodos refractarios de excitación del miocardio. Además puede actuar por otros mecanismos, entre los que cabe destacar la inducción de lesiones estructurales directas sobre el músculo cardíaco, el incremento de masa ventricular, sus efectos neurohormonales con elevación de la concentración de catecolaminas y la depleción de electrólitos que provoca. También es muy frecuente la inducción de arritmias durante los episodios de abstinencia alcohólica. De nuevo estos efectos arritmogénicos agudos del alcohol son mucho más frecuentes en pacientes alcohólicos crónicos, sobre todo en los afectos de cardiopatía subyacente más que en pacientes abstemios o sin cardiopatía. (6)

El alcohol ingerido en cantidades excesivas tiene un importante efecto tóxico sobre el corazón, no sólo sobre su capacidad de contracción sino también sobre el ritmo de los latidos. Un amplio estudio que incluye más de 15.000 personas ha confirmado que los sujetos que beben por encima de cuatro o cinco bebidas alcohólicas diarias tienen un $45 \%$ más de posibilidades de sufrir una arritmia cardiaca. (11) Hipertensión arterial.

Epidemiológicamente, el consumo de alcohol es un factor productor de hipertensión arterial muy frecuente (5-7\%). Aunque parece demostrado que más de dos copas al día se asocian con la hipertensión arterial (HTA), en los últimos tiempos se llega a afirmar que incluso ingestas menores podrían tener un efecto perjudicial desde este punto de vista. Se puede plantear con certeza que la bebida es un factor que aumenta claramente la probabilidad de incrementar la presión arterial. (12) 
El alcohol puede afectar la presión arterial de diferentes maneras. En primer lugar, el exceso de alcohol provoca un aumento en el peso, ya que el alcohol contiene un exceso de calorías. El exceso de las grasas causa un exceso de la presión en las arterias y, consecuentemente, en el corazón. Esto aumenta la presión en las arterias y en el corazón, indirectamente causando un aumento de la presión arterial. En segundo lugar, el exceso de alcohol, también tiene un efecto adverso sobre el funcionamiento de los riñones. Cuando los riñones están dañados, ejercen una presión sobre las arterias, dando lugar a la hipertensión. Los efectos del consumo de alcohol sobre la presión arterial difieren de una persona a otra. En algunas personas sólo puede darse un ligero aumento, mientras que en otras, puede haber un aumento drástico. Se observa, que los efectos del alcohol sobre la presión sanguínea son similares tanto en las mujeres como en los hombres. (12)

\section{Accidente vascular cerebral}

La relación entre consumo de alcohol y riesgo de AVC es compleja, ya que incluye beneficios y riesgos. De hecho, el consumo de alcohol no se interpreta como un riesgo mayor de AVC, sino como un factor de riesgo no bien definido. Por una parte, existe una clara relación entre consumo de alcohol y riesgo de AVC isquémico, siendo el consumo moderado de alcohol protector y el consumo elevado inductor de mayor riesgo. Este efecto protector en AVC isquémicos es más claro en hombres que en mujeres y probablemente el consumo de vino sería más protector que el de otras bebidas alcohólicas. En cambio, el riesgo de AVC hemorrágico aumenta progresivamente con el consumo de alcohol, sobre todo a partir de $60 \mathrm{~g}$ al día. $(13,14)$

\section{Miocardiopatía alcohólica}

Miocardiopatía alcohólica o cardiomiopatía alcohólica es un trastorno en el cual el consumo excesivo y habitual de alcohol debilita el músculo cardíaco. El corazón no puede bombear sangre eficientemente, por lo que a su vez se ven afectados los pulmones, el hígado, el cerebro y otros aparatos del organismo. (15)

La ingesta de alcohol en cantidades excesivas tiene un efecto directamente tóxico sobre las células del músculo cardíaco. La miocardiopatía alcohólica es una forma de miocardiopatía dilatada provocada por el consumo habitual y excesivo de alcohol. (15)

La miocardiopatía alcohólica hace que el músculo cardíaco debilitado no bombee de manera eficiente, llevando a que se presente insuficiencia cardíaca. En los casos graves, la falta de flujo 
sanguíneo afecta todas las partes del organismo, provocando daños en múltiples tejidos y órganos. Las complicaciones pueden ser: insuficiencia cardíaca congestiva y arritmias cardíacas, incluyendo las arritmias letales. (15)

Podemos dar por concluido que el consumo excesivo de alcohol, puede ocasionar miocardiopatía alcohólica (el corazón se dilata y deja de funcionar adecuadamente). También puede generar infartos al corazón. Su consumo regular eleva los niveles de sodio y calcio en la célula y puede producir hipertensión arterial. Cuando se reduce el consumo en personas con hipertensión se logra disminuir la enfermedad. La ingesta aguda de alcohol, puede producir arritmias graves en personas sanas.

\section{Referencias bibliográficas}

1. Barrett K, Barman S, Boitano S, Brook H. GANONG: Fisiología Médica. 23rd ed. Mexico: McGRAW-HILL; 2010.

2. Anatonia Humana. Conceptos Básicos del Sistema Cardiovascular. [Online]. [cited 2016 oct 26. Available from: http://www.anatomiahumana.ucv.cl/efi/modulo24.html.

3. Arredondo Bruce A, Risco Morales O. La acción cardioprotectora del uso moderado de alcohol. Revista Médica Electrónica. 2014 mar; 36(2).

4. Texas Heart Institute. Centro de Información Cardiovascular. [Online].; 2016 [cited 2016 oct 23. Available from: http://www.texasheart.org/HIC/Topics_Esp/HSmart/riskspan.cfm.

5. Lasdrogas.com. Alcohol. [Online].; 2013 [cited 2016 oct 23. Available from: http://lasdrogas.net/alcohol/.

6. Fernandez-Solá J. Consumo de alcohol y riesgo cardiovascular. Hipertensión y Riesgo Vascular. 2005 ene; 22(3).

7. Farber-Wilson J. Current Clinical Issues: should doctors prescribe alcohol to adults? Ann Intern Med. 2003; 139(7).

8. Mukamal K, Katherine M, Conigrave M, Mittleman M, Camargo C. Roles of Drinking Pattern and Type of Alcohol Consumed in Coronary Heart Disease in Men. The New England Journal of Medicine. 2003 ene; 348: p. 109-118. 
9. Raghav C. Regular drinking might explain the French paradox. BMJ. 2004 dec; 329(1308 ).

10. Montaner J. Alcohol e insuficiencia cardiaca. [Online].; 2006 [cited 2016 oct 26. Available from: http://www.consumer.es/web/es/salud/problemas_de_salud/2006/09/27/155803.php.

11. Marco J. El consumo excesivo de alcohol duplica el riesgo de arritmia. Corazón. [Online].; 2005 [cited oct 26. Available from: http://www.elmundo.es/elmundosalud/2005/09/13/corazon/1126602089.html.

12. Salud y Bienestar. Los efectos del alcohol sobre la presión sanguínea. [Online].; 2014 [cited 2016 oct 26. Available from: http://lasaludi.info/efectos-del-alcohol-sobre-la-presionsanguinea.html.

13. Jackson V. Alcohol consumption and mortality in men with preexisting cerebrovascular disease. Arch Intern Med. 2003 may; 163(10).

14. Reynolds K, Lewis B, Nolen J, Kinney G, Sathya B. Alcohol consumption and risk of stroke: a meta-analysis. JAMA. 2003 feb; 289(5).

15. Clínica DAM Madrid. Miocardiopatía alcohólica. [Online].; 2006 [cited 2016 oct 26. Available from: https://www.clinicadam.com/salud/5/000174.html. 